\title{
A Minimally Morphologically Destructive Approach for DNA Retrieval and Whole-Genome Shotgun Sequencing of Pinned Historic Dipteran Vector Species
}

\author{
Petra Korlević $\mathbb{D}^{1,2, *}$, Erica McAlister $\mathbb{D}^{3}$, Matthew Mayho $\mathbb{D}^{2}$, Alex Makunin $\mathbb{D}^{2}$, Paul Flicek $\mathbb{D}^{1}$, and \\ Mara K.N. Lawniczak $\mathbb{D}^{2, *}$ \\ ${ }^{1}$ European Molecular Biology Laboratory, European Bioinformatics Institute, Wellcome Genome Campus, Hinxton, Cambridge, United Kingdom \\ Wellcome Sanger Institute, Wellcome Genome Campus, Hinxton, Cambridge, United Kingdom \\ ${ }^{3}$ Department of Life Sciences, Natural History Museum, London, United Kingdom \\ *Corresponding authors: E-mails: korlevic@ebi.ac.uk; mara@sanger.ac.uk.
}

Accepted: 22 September 2021

\begin{abstract}
Museum collections contain enormous quantities of insect specimens collected over the past century, covering a period of increased and varied insecticide usage. These historic collections are therefore incredibly valuable as genomic snapshots of organisms before, during, and after exposure to novel selective pressures. However, these samples come with their own challenges compared with present-day collections, as they are fragile and retrievable DNA is low yield and fragmented. In this article, we tested several DNA extraction procedures across pinned historic Diptera specimens from four disease vector genera: Anopheles, Aedes, Culex, and Glossina. We identify an approach that minimizes morphological damage while maximizing DNA retrieval for Illumina library preparation and sequencing that can accommodate the fragmented and low yield nature of historic DNA. We identify several key points in retrieving sufficient DNA while keeping morphological damage to a minimum: an initial rehydration step, a short incubation without agitation in a modified low salt Proteinase K buffer (referred to as "lysis buffer C" throughout), and critical point drying of samples post-extraction to prevent tissue collapse caused by air drying. The suggested method presented here provides a solid foundation for exploring the genomes and morphology of historic Diptera collections.
\end{abstract}

Key words: Diptera, disease vectors, historic DNA, museum genomics.

\section{Significance}

Large museum collections of pinned insects could provide important snapshots of genomes through time, but unfortunately DNA retrieval from such fragile samples often leads to severe morphological damage, especially in delicate species such as disease transmitting Diptera. In this study, we have worked on a combined method that minimizes morphological damage while maximizing the retrieval of DNA from dry pinned Diptera species. We identified the importance of tissue rehydration, gentle DNA lysis buffer incubation, and critical point drying to restore collapsed tissues. We hope this approach will make it possible for more historic insect specimens to become available for genomic research while ensuring they remain intact for morphological studies.

\section{Introduction}

Over the past 100 years, heavy use of pesticides has resulted in novel evolutionary pressures on targeted species, often leading to successful control that is swiftly followed by resistance
(Forgash 1984; AL-Ahmadi 2019). For example, vector control measures such as insecticide-treated bednets and indoor residual spraying have had a positive impact on the control of human malaria vectors of the Anopheles genus (Kleinschmidt and

(C) The Author(s) 2021. Published by Oxford University Press on behalf of the Society for Molecular Biology and Evolution.

This is an Open Access article distributed under the terms of the Creative Commons Attribution License (https://creativecommons.org/licenses/by/4.0/), which permits unrestricted reuse, distribution, and reproduction in any medium, provided the original work is properly cited. 
Rowland 2019). However, this also caused an increase in insecticide resistance (Forgash 1984). Present-day populations of major malaria vector species from the Anopheles gambiae complex across sub-Saharan Africa now have widespread insecticide resistance (Kerah-Hinzoumbé et al. 2008; Edi et al. 2014; Clarkson et al. 2021; Munywoki et al. 2021), with similar increases observed in other malaria vectors, such as An. funestus (Riveron et al. 2015), An. stephensi (Yared et al. 2020), as well as other Dipteran disease vectors such as Aedes aegypti (Satoto et al. 2019), the vector responsible for transmitting yellow and dengue fever (Powell et al. 2018). Widespread insecticide use in sub-Saharan Africa started in the 1950s with DDT (dichlorodiphenyltrichloroethane) (Mendis et al. 2009), with new insecticides frequently introduced (Oxborough et al. 2015). Although mosquito genetic population structure change has been researched over the past 20 years (An. gambiae 1000 Genomes Consortium et al. 2017; Githeko et al. 1996; Gloria-Soria et al. 2016), it is unclear what population structure looked like prior to major vector control initiatives. Museum and other historic collections contain specimens preand post- the introduction of DDT and other insecticides, providing snapshots of populations that were reacting to these new evolutionary pressures in "real time." These collections could be used to fill gaps in our understanding about the evolution of insecticide resistance and study how historic populations compare with present-day genomic landscapes. Furthermore, historic collections also include the namebearing type specimens for these species and recovering genomic data from them could help us understand complex species evolution (Strutzenberger et al. 2012; Prosser et al. 2016).

Museum Diptera specimens are often identified prior to pinning, and due to improper mounting, storage, and general wear and tear from handling can accumulate morphological damage (Walker et al. 1999). On top of the samples themselves being quite fragile, the DNA from historic insect specimens is more fragmented and damaged than DNA from present-day individuals of the same species, and yields are usually lower. After the death of an organism DNA start degrading due to chemical processes such as hydrolysis and oxidation, which cause strands to break and accumulate base damage, the most common being cytosine deamination into uracil, particularly in single-stranded overhangs at the ends of molecules (Lindahl 1993). DNA in ancient samples such as fossil bones and teeth accumulated thousands of years of postmortem damage and the fraction of $5^{\prime} \mathrm{C}>\mathrm{T}$ and $3^{\prime}$ $G>A$ DNA substitutions resulting from cytosine deamination can reach $20-60 \%$ depending on age and species (Briggs et al. 2007; Dabney et al. 2013). Because of that, specific techniques have been developed for the retrieval, sequencing, and processing of ancient DNA, including recovery of ultrashort DNA fragments during extraction (Dabney et al. 2013; Rohland et al. 2018), optimization of double- or singlestranded library preparation and sequencing (Meyer and Kircher 2010; Briggs and Heyn 2012; Gansauge et al.
2020), as well as post-sequencing approaches to deal with high proportions of contaminant DNA and to make the most out of short, deaminated endogenous reads (Skoglund et al. 2014; Racimo et al. 2016; Link et al. 2017). Fortunately, historic samples had a shorter time frame for accumulating damage, are often stored in archives with stable temperatures and humidity levels limiting microbial growth and contaminant DNA accumulation, and although the retrieved endogenous DNA still tends to be very short, substitutions arising from cytosine deamination are much lower (about 2-5\%) (Bi et al. 2013; Weiß et al. 2016; Gutaker et al. 2017; Parejo et al. 2020), making it easier to account for them during data processing such as variant calling. Previous work on historic insect specimens have primarily focused either on complete destruction of individual specimens or parts of specimens followed by PCR or whole-genome sequencing (Parmakelis et al. 2008; Staats et al. 2013; Timmermans et al. 2016; Andrade Justi et al. 2021), or less destructive approaches and PCRbased methods (Gilbert et al. 2007; Santos et al. 2018), which can lead to very high failure rates due to the fragmented nature of older DNA, as well as amplification of contaminant DNA molecules that are much longer than the target endogenous DNA. Some papers combine a minimally destructive approach with whole-genome sequencing, but these were done on more robust insect species that can withstand harsher DNA lysis buffers (Tin et al. 2014; Parejo et al. 2020; Andrade Justi et al. 2021).

In this article, we present a minimally morphologically destructive approach for DNA retrieval from pinned historic vector Diptera specimens with the goal of maximizing DNA retrieval while minimizing irreversible morphological damage to precious specimens. Specimens were selected from the London Natural History Museum (NHM) Diptera collection. We focused primarily on sub-Saharan African malaria transmitting Anopheles mosquitoes and confirmed the range and efficacy on Aedes, Culex, and Glossina. We couple this with ancient DNA purification techniques, library preparation optimized for low yield extracts with short inserts, and processing the sequencing data using ancient DNA pipelines. A schematic of the initial steps, from selecting, cataloging, extracting DNA, purification, and returning the specimens to the collection is summarized in supplementary figure S1, Supplementary Material online. We show that by using this approach, it is possible to retrieve nuclear data and consensus mitochondrial genomes with shallow shotgun sequencing.

\section{Results}

DNA Retrieval from An. gambiae Complex Mosquitoes within the Last Century

As there has been limited genomic work on historic Anopheles specimens (Parmakelis et al. 2008; Andrade Justi et al. 2021), we wanted to evaluate yields and ancient DNA 
characteristics in mosquitoes collected within the past century, as well as the stability of their morphological integrity during handling. For this, we selected several major and minor vector species from the An. gambiae complex spread across six decades (1930s-1980s) (specimen metadata in supplementary table S1, Supplementary Material online). This initial approach included rehydrating pinned samples prior to submerging them in "lysis buffer $A$," adapted from a recently published low salt Proteinase K tissue clarifying buffer for preparing samples for microscopy (Santos et al. 2018). After overnight incubation, the specimens were rinsed with ethanol and air dried, whereas the lysis buffer was purified using a modified MinElute silica column approach used in ancient DNA research (Dabney et al. 2013). We noticed the rehydration step was crucial in order to minimize damage caused by static electricity, as samples after rehydration only occasionally lost legs or rarely the head, which was primarily due to the original placement of the specimen pin. We also noticed early on that air drying post extraction was not suitable, as very fine structures such as abdomens, limbs, and antennae collapsed. To counteract this, the samples were taken through a series of ethanol concentrations from $30 \%$ to $100 \%$, and then critical point dried (CPD) with liquid $\mathrm{CO}_{2}$. This procedure, although laborious, greatly improved morphological characteristic accessibility (supplementary fig. S2, Supplementary Material online).

In terms of DNA retrieval we had a wide range of estimated DNA yields (determined using a Quant-iT PicoGreen dsDNA Assay Kit), from only $3 \mathrm{ng}$ for a specimen collected in the 1930s (value similar to our extraction blanks) to nearly $200 \mathrm{ng}$ from 1980s specimens (fig. 1A and supplementary table S3, Supplementary Material online), with much longer DNA fragments also present in the more recently collected specimens as assessed by an Agilent Bioanalyzer High Sensitivity DNA Analysis Chip (supplementary fig. S3, Supplementary Material online). These are estimates of the total DNA retrieved from each specimen, and are affected by coextracted non-DNA molecules, a mixture of double- and single-stranded fragments, and could be microbial growth post-pinning.

Due to the fragmented and low yield nature of these DNA extracts, we had to adapt our double-stranded library approach (Bronner and Quail 2019) to fit this type of input compared with present-day samples. Initial extract volumes were split into two library preparation strategies for each sample. For older samples that contained DNA below 300 bp based on Agilent chips, the post-ligation libraries were purified either with SPRI beads or silica columns. For younger samples that contained DNA up to $10,000 \mathrm{bp}$, the extract was split in half with one half being sheared and the other going into library preparation unsheared. Libraries were amplified by indexing PCR which introduced 8 bp tags on both ends, pooled and sequenced using a 75 paired-end (PE) approach on a lane of an Illumina HiSeq 2500 system (pool containing 20 sample libraries with a lower fraction for four extraction blank libraries). Libraries were then processed using the ancient DNA pipeline EAGER (Fellows Yates et al. 2021) as well as tools already integrated in samtools (Li et al. 2009).

The summary statistics after mapping to the An. gambiae reference genome (AgamP4) are shown in figure 1B-D. Several specimens investigated here are from species in the An. gambiae complex that are substantially diverged from the An. gambiae s.s. reference. Therefore, lower percentages of aligning reads could be driven by this divergence and/or nonendogenous DNA such as DNA from microbial growth. In this initial assessment, we see that the percentage of DNA aligning to the reference varies in samples of more distantly related species (An. melas, An. merus), likely due to reduced similarity to the reference instead of an increased level of microbial contamination (Fontaine et al. 2015). For species more closely related to the AgamP4 reference (An. gambiae, An. arabiensis), we see a much higher fraction of sequences aligning, with older samples (collected further back in time) showing slightly lower nuclear coverage compared with younger samples of the same species. We also see that the different approaches during library preparation are virtually indistinguishable (circles vs diamonds in fig. 1), so for library preparation throughout the rest of this study we opted for the most parsimonious protocol of no shearing along with bead purification prior to indexing PCR in order to minimize the number of steps and simplify multichannel or robot work by avoiding individual sample tubes.

As we expect our library DNA inserts to be short, we merged overlapping 75 bp PE reads (with a minimum 11 bp overlap), and retained both merged (inserts $\leq 139 \mathrm{bp}$ ) and unmerged paired reads (inserts $>139 \mathrm{bp}$ ). When looking at the size distribution of aligned reads, we notice that the fraction of unmerged $(75 \mathrm{bp})$ reads increases with age, going all the way up to $50-60 \%$ of total reads for samples from the 1980s, whereas the oldest samples contain inserts that are on average only 40-60 bp long (supplementary fig. S4A and table S3, Supplementary Material online). Through this set we also clearly illustrated the need to use the correct polymerase to PCR amplify the libraries post-ligation, as our $5^{\prime} \mathrm{C}>\mathrm{T}$ signals were affected by a polymerase that could not recognize uracils, the incidence of which was estimated to about 3-5\% at molecule ends and $1-2 \%$ within the DNA fragments (supplementary fig. $S 4 B$ and $C$, Supplementary Material online). The opposite strand's $3^{\prime} \mathrm{G}>\mathrm{A}$ signal is not as affected because the pairing of adenines to uracils is done during library preparation.

\section{Maximizing DNA Retrieval while Minimizing Morphological Damage}

Our second experiment focused on the retrieval of DNA and the level of morphological damage using three different lysis buffers (extensively tested on present-day Anopheles 

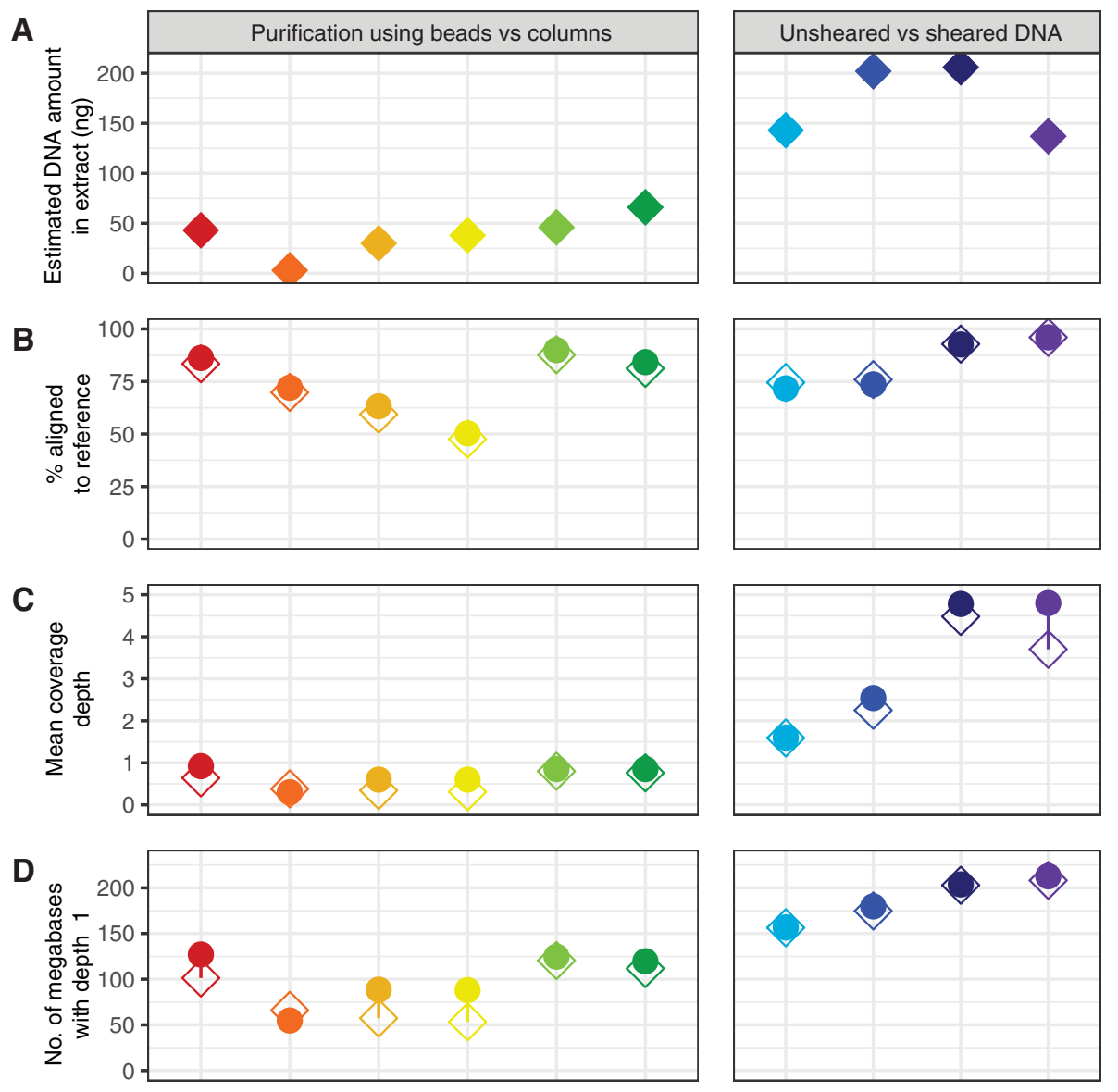

FIG. 1.-Sequencing data summary of ten historic Anopheles gambiae complex specimens. For older samples (left and middle panels, 1930s-1960s) libraries post-ligation were split in half and purified using SPRI beads or MinElute silica columns, whereas for younger samples (right panels, 1980s) DNA extracts were split in half, one half was sheared, and the unsheared and sheared aliquots underwent library preparation separately. (A) Estimated DNA yields in nanograms (ng) measured by Quant-iT PicoGreen dsDNA Assay Kit. (B) Percentage of sequences in each library aligning to the AgamP4 An. gambiae reference. Variation can be driven by nonendogenous DNA and/or by aligning to a mismatched reference genome. (C) Mean nuclear depth of coverage achieved for each library. $(D)$ Number of megabases in the AgamP4 reference genome covered with a depth of at least $1 \times$ or more (maximum $230,466,657$ ).

mosquitoes and outlined in Makunin et al. [2021]) on An. gambiae, An. melas, and An. funestus samples at the oldest age range of historic collections we are aware of (1920s1940s) (specimen metadata in supplementary table S1, Supplementary Material online). Samples were initially incubated overnight, and we observed substantial levels of clarification and pigment loss, especially in smaller species such as An. funestus (supplementary fig. S12, Supplementary Material online), so a smaller subset of An. funestus specimens were instead incubated for only $2 \mathrm{~h}$. Unfortunately, we could not perform a thorough assessment of morphology as the CPD instrument malfunctioned, and there was substantial damage caused by prolonged exposure to high concentrations of ethanol as well as insufficient $\mathrm{CO}_{2}$ during the drying procedure. However, it appeared that a $2 \mathrm{~h}$ incubation caused less damage and internal tissue loss (including blood meals) compared with an overnight incubation (supplementary fig. S13, Supplementary Material online).
Comparable to what was observed in present-day samples (Makunin et al. 2021), there are no clear differences in total DNA yields between the three buffers (fig. $2 A$ and supplementary fig. S6 and table S3, Supplementary Material online). It is far more likely that the observed variation in DNA retrieval is due to within-sample set variation, and not caused by the buffers themselves, though we have tried minimizing such variation by selecting samples collected from similar times and locations. Lysis buffer $C$ and $G$ containing no Proteinase $\mathrm{K}$ resulted in the lowest yields but a similar level of morphological damage, so Proteinase $\mathrm{K}$ was used in all further testing of these two buffers.

Libraries were prepared following the simplified approach described above (no shearing, lower concentration SPRI purifications post-ligation and post-P(R), and samples were pooled into three pools (An. gambiae/An. melas pool 24 samples +9 blanks, An. funestus overnight pool 18 samples +3 blanks, An. funestus $2 \mathrm{~h}$ pool 20 samples +3 blanks; not all 


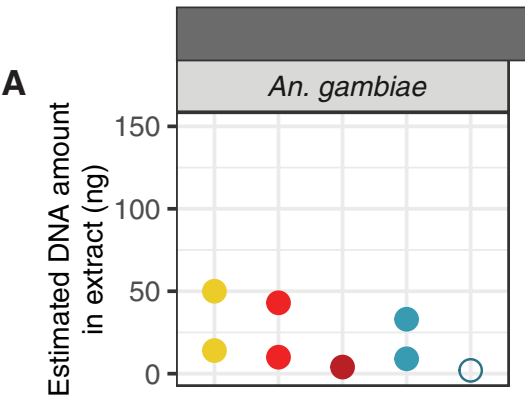

\section{Overnight incubation}
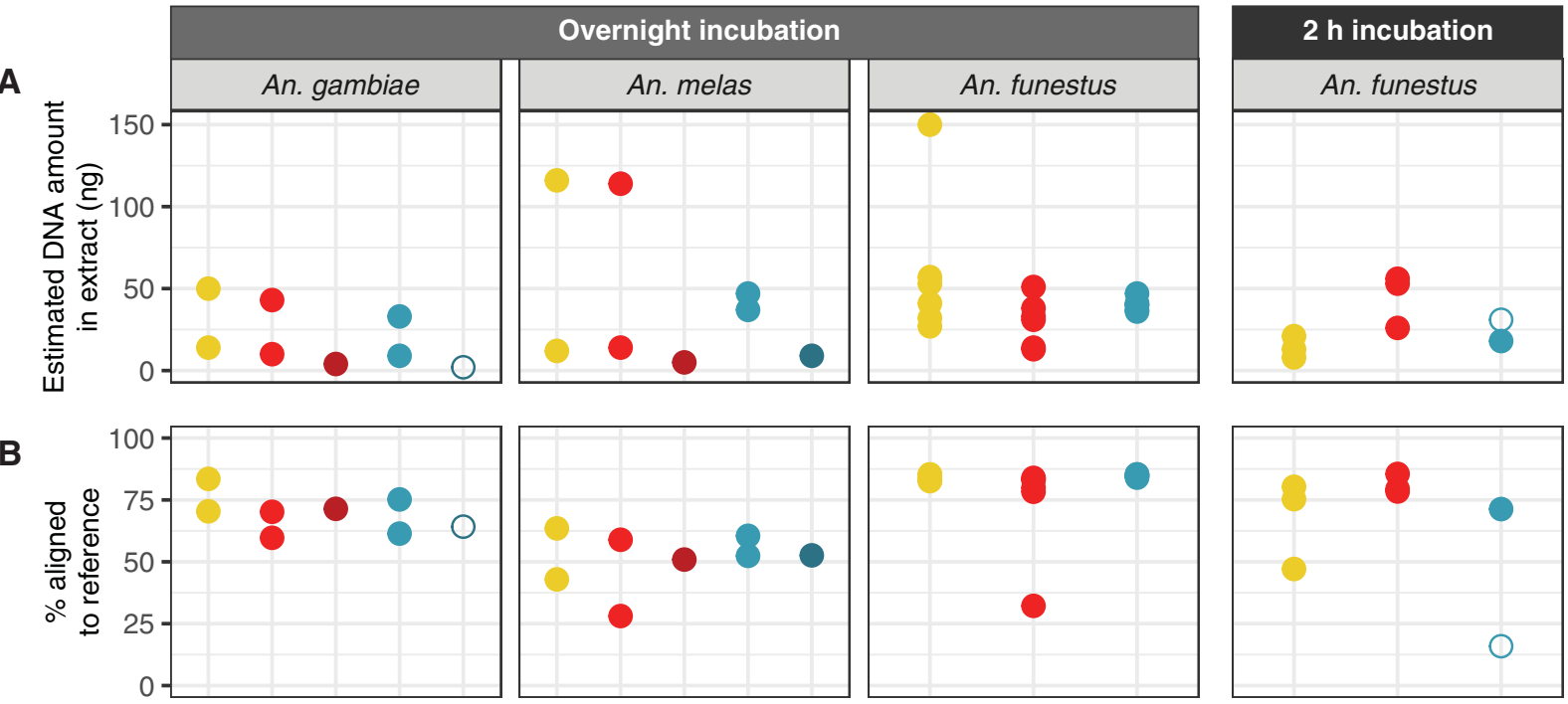

C
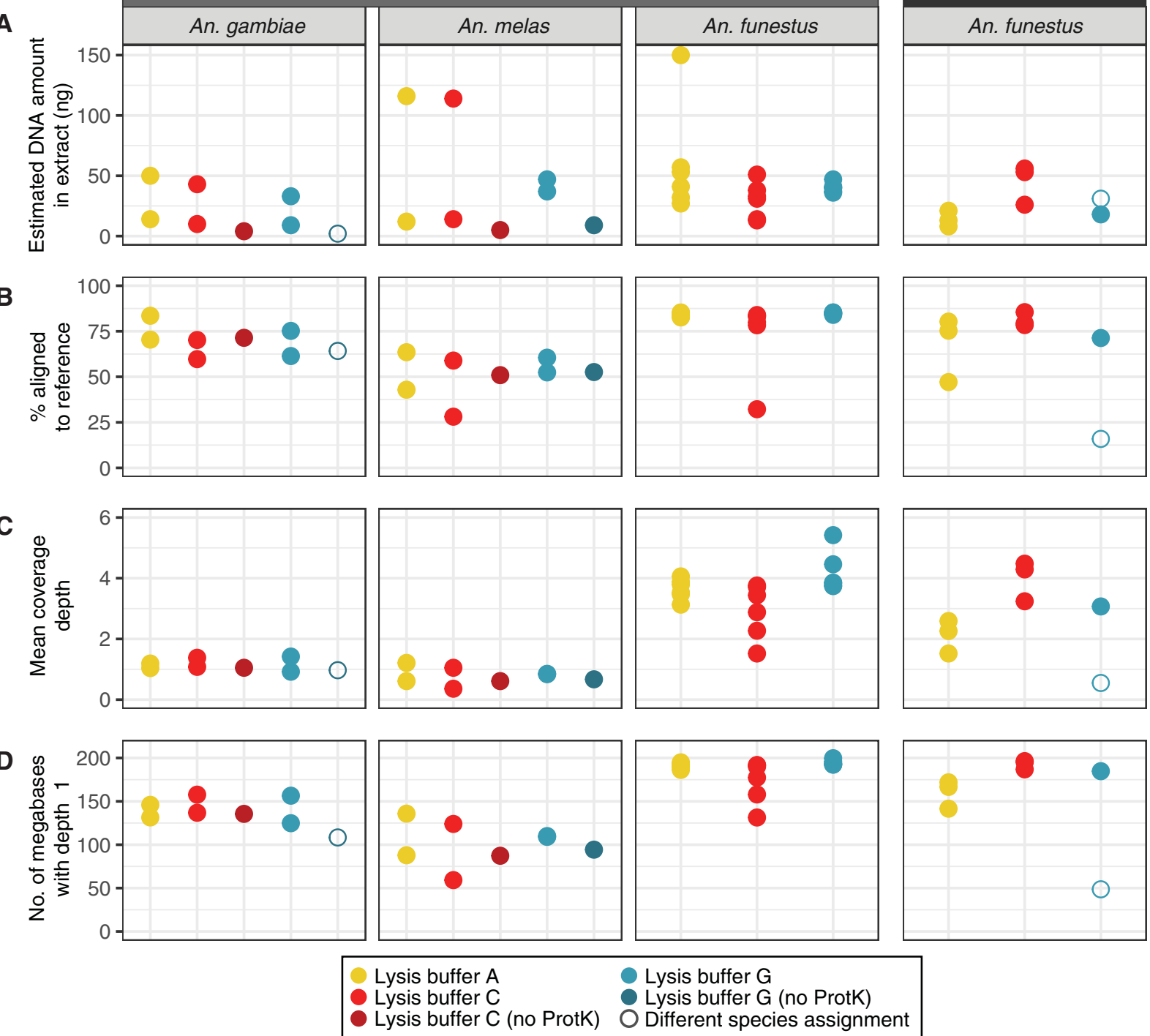

$$
\begin{aligned}
& \text { Lysis buffer } \mathrm{G} \\
& \text { Lysis buffer } \mathrm{G} \text { (no ProtK) } \\
& \text { Different species assignment }
\end{aligned}
$$

FIG. 2.-Sequencing data summary of overnight incubated Anopheles gambiae $(n=8)$, overnight incubated An. melas $(n=8)$, and overnight or $2 \mathrm{~h}$ incubated $A n$. funestus $(n=26)$ specimens in three different lysis buffers, two of which were also tested when they contained no Proteinase K. (A) Estimated DNA yields in nanograms (ng) measured by Quant-iT PicoGreen dsDNA Assay Kit. (B) Percentage of sequences in each library aligning to An. gambiae (AgamP4) or An. funestus (AfunF3) reference. (C) Mean nuclear coverage depth in each library. (D) Number of megabases in the AgamP4 reference covered with a depth of at least $1 \times$ or more (maximum 230,466,657) for An. gambiae and An. melas, or AfunF3 (maximum 210,975,322) for An. funestus. Samples represented with empty circles were found to be a different species after sequencing and mitochondrial assembly (An. funestus mapped to AfunF3 in the first column, An. rivulorum in the last column).

libraries from the first and last pool are discussed here as they are part of another project). The pools were sequenced on three lanes of an Illumina HiSeq 400075 PE with the same processing and summary statistics generation as for our first experiment, An. gambiae/An. melas libraries were mapped to AgamP4, and the An. funestus libraries were mapped to the An. funestus nuclear (AfunF3) and mitochondrial (NC_038158.1) references.

After sequencing we noticed a similar picture to our first sample set, with $70 \%$ of reads in An. gambiae samples and
$51 \%$ of reads in An. melas samples on average mapping to the AgamP4 reference, whereas An. funestus samples on average had $79 \%$ of reads mapping to the AfunF3 reference (fig. 2B and supplementary table S3, Supplementary Material online). In this set we also found two samples, NHMUK010633485 previously determined as An. gambiae, and NHMUK013655440 previously determined as An. funestus, for which their original morphological assessment and mitochondrial genome-based assessment did not match. Mitochondrial data from these specimens grouped with 
mitochondrial genomes most similar to An. funestus and $A n$. rivulorum, respectively (supplementary fig. S9, Supplementary Material online). The first sample was therefore mapped to AfunF3 and NC_038158.1, whereas unfortunately for An. rivulorum, we do not have a more appropriate reference. Comparing the overnight and $2 \mathrm{~h}$ incubations for the $A n$. funestus specimens, we retrieved a substantial amount of mosquito DNA after a $2 \mathrm{~h}$ incubation compared with overnight (similar total yields, percent aligned, and just slightly lower coverage) (fig. $2 B-D$ ), confirming a $2 \mathrm{~h}$ incubation is better for maximizing DNA retrieval from pinned specimens while minimizing morphological damage. We also see no substantial difference between buffers in terms of deamination patterns or retrieval of longer or shorter DNA sequences (supplementary fig. S7, Supplementary Material online). The difference in length observed for An. funestus samples, with plenty of inserts being too long to overlap and a prominent unmerged ( $75 \mathrm{bp}$ ) peak, is likely due to the different strategy of SPRI bead purification post-ligation (one round of $2.2 \times$ SPRI compared with $2.5 \times$ SPRI used for the An. gambiae complex samples) and post-indexing PCR (two rounds of $1 \times$ SPRI compared with a single round of $1.2 \times$ SPRI) (supplementary table S3, Supplementary Material online). This larger portion of longer molecules, combined with a lower pool plex, likely explains why we obtain higher coverage for An. funestus samples compared with the An. gambiae complex samples (fig. 2C), and so a slight selection against very short molecules in samples of these decades and younger, coupled with a plex level of under 20 samples per pool, could be beneficial to lower sequencing costs.

\section{Efficiency across Different Vector Diptera Species}

In our final experiment, we focused on what we believe is currently the best approach for simultaneously retrieving adequate amounts of DNA for further genomic work and minimizing morphological damage to the pinned dry specimen based on both our findings and current literature: rehydrating the pinned specimens prior to handling for $3 \mathrm{~h}$ at $37^{\circ} \mathrm{C}$, incubating in lysis buffer for $2 \mathrm{~h}$ at $37^{\circ} \mathrm{C}$, rinsing the specimens in $30 \%$ ethanol and storing in 50\% ethanol prior to full ethanol dehydration series and CPD. For this we selected four Dipteran vector species: three mosquitoes Aedes, Anopheles, Culex, and one tsetse fly Glossina (specimen metadata in supplementary table S1, Supplementary Material online). Specimens from each genus were selected across three decades (1930s, 1950s, and 1970s) with the idea of assessing differences in DNA retrieval and morphological damage caused to samples of different ages. However, there was substantial variation within and between these species/decade sets, with samples from the 1930s and 1950s occasionally containing longer DNA and higher yields than samples from the 1970s (supplementary table S3 and fig. S10, Supplementary Material online). This is likely due to initial specimen handling at collection and storage prior to being archived at the NHM, information which is very often missing from the pinned specimen labels.

A detailed evaluation of morphological changes post DNA extraction and CPD, as well as our internal score system detailing what level of damage we count as "pass" (key diagnostic features still preserved) or "fail," is outlined in supplementary table S2, Supplementary Material online. We noticed that the lowest level of morphological damage across all species was obtained with lysis buffer C (11 pass, 1 fail), with buffer A performing slightly worse (8 pass, 3 fail), and buffer $\mathrm{G}$ performing the worst ( 5 pass, 7 fail) (representative specimens across genera for each buffer showcased in supplementary figs. S14-S17, Supplementary Material online, with a closer look at an Anopheles lysis buffer C "pass" specimen in fig. 3A). When looking across the different genera regardless of lysis buffer, the most affected were Anopheles (3 pass, 5 fail), followed by Aedes ( 5 pass, 4 fail) and Culex (7 pass, 2 fail), whereas all nine Glossina specimens got a passing score.

In terms of DNA retrieval, we noticed a similar release between the three buffers across all species, with slightly more DNA retrieved from Aedes and Culex compared with Anopheles and surprisingly Glossina, which had very low DNA yields for their size (fig. 3B). This might be due to extensive soft tissue degradation in the abdomen and thorax (specimens appeared to be hollow). It is also possible that larger samples such as Glossina require longer incubation times than $2 \mathrm{~h}$ in order for the buffer to penetrate deeper through the outer chitin layer. DNA length was also very variable between different species, decades, and lysis buffers (supplementary fig. S10, Supplementary Material online).

\section{Discussion}

Historic museum collections are invaluable snapshots through space and time of populations that lived in a period prior to, during, and directly after extreme anthropogenic influences, such as the widespread use of insecticides in order to control populations of disease vectors (Forgash 1984; Kleinschmidt and Rowland 2019). With ever evolving methods for the retrieval and sequencing of old, fragmented and low yield DNA, primarily for ancient DNA research of fossil bones and teeth, we now can also apply and modify such methods to obtain genomic data from historic specimens (Staats et al. 2013; Gutaker et al. 2017; Parejo et al. 2020; Andrade Justi et al. 2021). In this article, we have focused on important human disease vectors, especially sub-Saharan malaria transmitting Anopheles mosquitoes. Over the course of three experimental setups, we have evaluated the amount of DNA that can be retrieved from dried and pinned historic specimens seeking approaches that minimize the level of morphological damage caused during sample handling while maximizing the amount of informative genomic data we can obtain. 


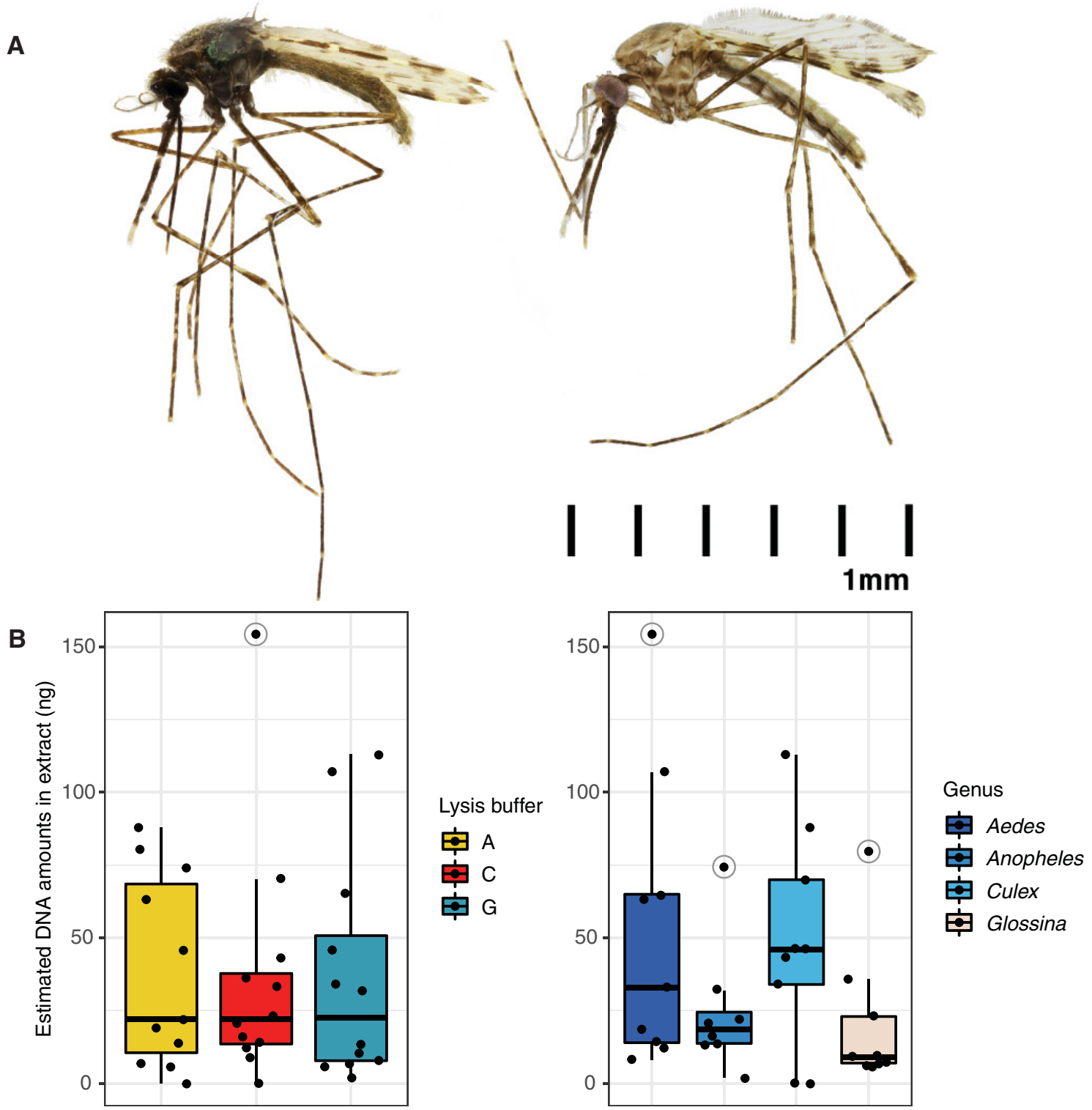

FIG. 3.-Lysis buffer efficiency across four Dipteran vectors. (A) Focus stacked images of NHMUK010633504 (Anopheles) before (left) and after (right) a $2 \mathrm{~h}$ DNA extraction with lysis buffer $\mathrm{C}$. This specimen sustained minimal morphological alterations after extraction. Adobe Photoshop was used for normalizing brightness and contrast, resizing (with millimeter scale attached), rotating and removing backgrounds. An overview of other representative specimens from all four genera and all three buffers is presented in supplementary table S2 and figures S14-S17, Supplementary Material online. (B) Estimated DNA yields in nanograms (ng) measured by Quant-iT PicoGreen dsDNA Assay Kit. Left is grouped by buffer and right is grouped by genus. The individual sample data points are represented by black dots with outliers marked using gray circles.

Across all of our experiments we have identified key points that are crucial in minimizing the level of morphological damage afflicted to the specimen during DNA extraction. These include rehydration of desiccated tissues, a very short lysis $(2 \mathrm{~h})$, and CPD soon after the lysis is performed to minimize damage from conventional air drying or prolonged exposure to ethanol. We also noticed that the initial mounting procedures, including the thickness of pins that pierce the specimen's thorax and general pin placement, affects contact with liquids or plasticware that may degrade the specimen's morphology. One of the biggest risks we identified was that if pins were placed too high up in the thorax, it led to decapitation or neck extension (supplementary fig. S2, Supplementary Material online). Fortunately, any larger body pieces such as limbs, the head or abdomen, can be collected and stored in capsules together with the specimen's body because, although the specimen is no longer intact, no tissue is mechanically destroyed by grinding during the extraction procedure. 
We have also noticed that some surface structures on certain species tend to be more prone to damage during the extraction and drying procedure, such as bristles on the head and thorax, as those were lost across nearly all species presented in this article (supplementary table S2, Supplementary Material online). However, other key morphological features, such as scales across the body and limbs, including wings, were largely unaffected in all samples. Furthermore, even though $2 \mathrm{~h}$ seemed to work perfectly fine for most, shorter or longer incubation times might be required for some species, as seen for the very low DNA release for physically much larger Glossina samples.

In terms of DNA retrieval, we saw a lower total DNA yield compared with present-day samples extracted across all three used buffers, which was expected due to degradation of DNA through time (supplementary fig. S11, Supplementary Material online, present-day data from Makunin et al. [2021]). Our samples were at the lower end of DNA yields compared with present-day samples (yields of $43 \pm 43 \mathrm{ng}$ on average across all buffers and incubation times compared with $172 \pm 99 \mathrm{ng}$ for present-day samples), as well as severely fragmented in most cases (supplementary figs. S3, S6, and S10, Supplementary Material online). Because of that we modified our library preparation to accommodate for such short low yield inserts and noticed that less stringent library preparation approaches can be used compared with regular ancient DNA libraries. To make processing historic samples faster we opted for no shearing for samples that still contain slightly longer DNA, as well as SPRI bead purification postligation instead of column cleanups, the concentrations of which were adapted to fit a plate setup ( $2.2 \times$ post-ligation, two rounds of $1 \times$ post-indexing P(R). We have also shown that typical ancient DNA damage patterns are present in these samples, although as expected for DNA that is only decades old, at a much lower rate (up to $5 \% \mathrm{C}>\mathrm{T}$ at the $5^{\prime}$ end and $\mathrm{G}>\mathrm{A}$ at the $3^{\prime}$ end, with $1-2 \%$ in the middle of the molecules, similar to what is observed for other historic tissues such as herbarium collections [Gutaker et al. 2017]) (supplementary figs. S4 and S7, Supplementary Material online).

Our plex levels and sequencing depths (18-24 libraries on a single 75 PE HiSeq 2500 or 4000 lane, expected yields per lane 41 and $43.75 \mathrm{~Gb}$, respectively, with insert sizes often below $150 \mathrm{bp}$ ) vary substantially from what is typically used for obtaining 30x coverage for present-day Anopheles specimens (36 libraries on three 150 PE HiSeq X10 lanes, expected yield for all three lanes is $330 \mathrm{~Gb}$, with insert sizes often above $300 \mathrm{bp}$ ). Although on average we retrieved about $2.6 \times$ mean nuclear coverage depth (from 0.4 to $9.3 \times$ ), we were also able to retrieve $372 \times$ mean mitochondrial coverage (from 10 to $1,583 \times$ ), and could assemble consensus mitochondrial genomes for all samples, including very low yield samples extracted with lysis buffers without any Proteinase K. Additionally, our historic samples have higher complexity than typical ancient DNA libraries, and the over-sequencing of PCR duplicates is fairly low (average duplication rate of $0.12 \pm 0.05$ across sample libraries, supplementary table S3, Supplementary Material online). This means there are still plenty of unique sequences present in each library, and additional sequencing can be performed to reach coverage levels of 20-30x which will facilitate genotyping and minimize potential biases caused by ancient DNA substitutions. As for the consensus mitochondrial genomes, we compared them with $\mathrm{NCBI}$ available genomes, some of which were published mitochondrial phylogenies of species in the An. gambiae complex (Beard et al. 1993; Peng et al. 2016; Hanemaaijer et al. 2018) and An. funestus complex (Hua et al. 2016; Peng et al. 2016; Jones et al. 2018; Liu et al. 2019; Small et al. 2020), and created maximum likelihood trees using MAFFT and FastTree incorporating both present-day samples and historic specimens (supplementary figs. S8 and 59, Supplementary Material online). This helped in assessing that two samples were misidentified, and one could be mapped to the proper reference. The potential of creating mitochondrial genomes could help immensely with categorizing misidentified specimens in collections, especially species with less prominent vertical gene transfer and hybridization potential as malaria transmitting Anopheles. Even though low coverage, we also checked known insecticide resistance variants in present-day An. gambiae complex populations in the voltage-gated sodium channel gene (Clarkson et al. 2021), and found no evidence of the emergence of known insecticide resistance variants in our sequenced specimens (supplementary table S4, Supplementary Material online). However, our data set requires deeper sequencing and sequencing of more specimens to have the necessary resolution to assess the origin of insecticide resistance variants in more detail.

Across all our experiments, we have noticed minimal difference between the three lysis buffers used, however lysis buffer $C$ slightly outperforms the others when looking at the level of morphological damage after DNA extraction. As mosquitoes are quite fragile compared with other species of flies, more work is required in order to get a better understanding on what are the ideal extraction conditions (temperature, time) for different species to minimize morphological damage even further. We recommend doing an initial DNA extraction test on present-day samples (as already highlighted for Anopheles specimens in Makunin et al. [2021]) to assess the level of morphological damage that might be caused by handling and exposure to lysis buffer, especially noting down changes in morphologically relevant traits such as proteinbased pigments, which will likely be destroyed with proteinase based lysis buffers (Santos et al. 2018). A very important part of the process would also be the selection of samples from the start, as poorly preserved samples are likely to be further damaged in the whole process. Other optimizations could include streamlining the DNA purification process by using silica beads instead of columns, so the whole procedure 
could be performed in plates instead of single tubes (Rohland et al. 2018).

Given the shrinking community of traditional taxonomic expertise, together with the challenge for many insects in confidently "getting to species" using morphology alone, we hope that our approach will enable increased momentum to genetically characterize museum collections where huge amounts of time and effort have been spent identifying and maintaining these valuable archives. Beyond linking genetic identities with taxonomically identified species, genetic characterization of museum specimens will also help with elucidating population structure changes over the past century, particularly in insect species such as disease-transmitting Diptera or those most affected by the climate crisis. We anticipate that over the coming years, with improved genomic access to museum specimens driven by approaches like the one we developed here, we will be able to study the changes observed in the genomes of present-day individuals in real time across the last century, while also preserving precious and limited historic pinned samples for future generations.

\section{Materials and Methods}

\section{Historic Pinned Specimen Selection}

Across all experiments, we retrieved DNA from 87 pinned dry Diptera specimens from the NHM's 2.5 million specimen Diptera collection (verbatim labels in supplementary table S1, Supplementary Material online). Based on the labels accompanying each specimen, these were classified as An. gambiae $(n=20)$, An. melas $(n=10)$, An. merus $(n=2)$, An. arabiensis $(n=2)$, An. funestus $(n=26)$, Aedes aegypti $(n=9)$, Culex pipiens $(n=9)$, and Glossina morsitans $(n=9)$. The specimens were collected across a wide range of years and locations, from 1927 to 1988 and spanning 14 countries. Specimens were imaged both before and after DNA extraction using a Canon 5DSR with an Mp-E $65 \mathrm{~mm}$ stackshot rail for stacking. The wedge lights and specimen platform were custom built by the engineering department at the NHM. Eos Utility V.3, Helicon Remote, and Helicon Focus were used to create focus stacked images. Images were also taken at the Wellcome Genome Campus using a Hirox 3D digital microscope (before extraction photos of a few representative specimens in supplementary fig. S12, Supplementary Material online).

When working with historic specimens, similar to other ancient DNA work, it is critical to minimize the effects of modern day contaminants. Therefore, pinned historic Diptera samples were handled in laboratories where no present-day Diptera work was performed, especially avoiding post-PCR areas. During DNA extraction and purification, buffers were prepared and handled inside UV decontaminated PCR cabinets, and most reagents (besides SDS and Proteinase $\mathrm{K}$ ) and all DNA LoBind plasticware were decontaminated in a
UV crosslinker $2 \times 45$ min prior to use. Aliquots of purified DNA extracts were then transferred to post-PCR areas for quality control (concentration and fragment length measurements) as well as library preparation and sequencing. For each set, we included several extraction blanks (tubes containing no sample DNA, just buffers) which were processed the same way and sequenced in the same pools at a lower fraction (supplementary table S3, Supplementary Material online). We found no concerning sign of contamination with present-day DNA or cross-contamination with historic DNA in any of our blanks. Raw sequencing data for all specimen and blank libraries has been deposited in the European Nucleotide Archive (ENA) under study accession ERP129396, FASTQ IDs specified next to their corresponding NHM IDs in supplementary table S3, Supplementary Material online.

\section{Initial Assessment of DNA Preservation in Historic Anopheles}

First we tested the efficiency of DNA retrieval from ten pinned historic An. gambiae complex specimens using a minimally destructive low salt Proteinase $\mathrm{K}$ buffer described for insect tissue clarification prior to microscopy (consisting of $200 \mathrm{mM}$ Tris pH 8.0, $25 \mathrm{mM}$ EDTA pH 8.0, $250 \mathrm{mM} \mathrm{NaCl}, 0.5 \%$ SDS and $0.4 \mathrm{mg} / \mathrm{ml}$ Proteinase $\mathrm{K}$ as described in Santos et al. [2018]), in this article defined as "Iysis buffer A." Two samples at the most extreme ages (1938 and 1988) were removed from their label pins but left on their sample pins, placed into $2.0 \mathrm{ml}$ DNA LoBind tubes and $200 \mu \mathrm{l}$ of buffer A was added to completely submerge the sample. However, due to plasticware static electricity, the samples were torn apart during handling. Therefore, for the remaining eight samples we performed tissue rehydration for $3 \mathrm{~h}$ at $37^{\circ} \mathrm{C}$ in a styrofoam box containing wet paper towels prior to DNA lysis. After rehydration, samples were removed from their label pins and submerged into $200 \mu \mathrm{l}$ of buffer A. All samples regardless of rehydration were then incubated in the buffer at $37^{\circ} \mathrm{C}$ overnight. The next day, lysis buffer was transferred into new tubes, whereas samples were rinsed with $500 \mu \mathrm{l} 100 \%$ ethanol for $30 \mathrm{~min}$ and then air dried before returning to the NHM. We observed substantial tissue collapse caused by air drying (especially eyes, abdomens, and antennae), which led us to evaluate CPD with liquid $\mathrm{CO}_{2}$ to restore volume. Airdried samples were rehydrated in $30 \%$ ethanol, and a serial ethanol dehydration was performed $(20 \mathrm{~min}$ incubation in 30-50-70-90-3×100\% ethanol) followed by CPD on a Baltec CPD 030, which successfully restored volume to collapsed tissues (supplementary fig. S2, Supplementary Material online).

The lysates were purified using a MinElute PCR Purification Kit silica column approach optimized for the purification of an EDTA-rich lysis buffer used in DNA extraction from ancient bones and teeth (Dabney et al. 2013) with a few modifications. We added $200 \mu$ of lysis buffer A to $2.0 \mathrm{ml}$ DNA LoBind 
tubes containing UV-treated $1.4 \mathrm{ml}$ Qiagen binding buffer (PB) and $55 \mu \mathrm{l} 3 \mathrm{M}$ sodium acetate $(7 \times$ the buffer volume instead of the $5 \times$ volume recommended in the manufacturer's protocol or the $10 \times$ volume recommended by Dabney et al. [2013]). After the full volume of lysis buffer, PB and sodium acetate mixture was centrifuged through, columns were washed twice with $750 \mu$ l Qiagen wash buffer $(\mathrm{PE})$, dry spinned at maximum speed, and the elution of silica bound DNA was performed twice with $25 \mu$ of TET buffer (10 mM Tris pH 8.0, 1 mM EDTA pH 8.0, 0.05\% Tween-20) for a total eluate volume of $50 \mu \mathrm{l}$ stored in $1.5 \mathrm{ml}$ DNA LoBind tubes. Quality control of the final extracts was performed using a Quant-iT PicoGreen dsDNA Assay Kit for concentration estimates (supplementary table S3, Supplementary Material online), and an Agilent Bioanalyzer High Sensitivity DNA Analysis chip for concentration and DNA fragment length measurements prior to library preparation (supplementary fig. S3, Supplementary Material online). In total, $14 \mu$ l of the full extract volume was used for library preparation for the majority of samples, whereas for lower yield samples (NHMUK012805007, extraction blanks) $28 \mu \mathrm{l}$ was used instead.

\section{Adapting Library Preparation and Sequencing for Low Yield Short DNA Inserts}

For simplicity and easier streamlining, we modified the NEBNext Ultra II DNA Library Prep Kit for Illumina, a single tube double-stranded sequencing library preparation kit already optimized and widely used at the Wellcome Genome Campus sequencing facilities (Bronner and Quail 2019), for low yield short insert DNA extracts. Based on the initial quality control evaluation, six samples (1930s-1960s) had lower yields and short DNA, whereas four samples from the 1980s had higher yields and longer DNA. For the 1980s extracts at the start of library preparation the volume was split in half, with one half undergoing Covaris shearing prior to library preparation, and the other going into library preparation without shearing. For all other extracts the post-adapter ligation reaction was split in half, one half was purified using silica columns (MinElute PCR Purification Kit) and the other using $3 \times$ SPRI beads (Beckman Coulter Agencourt AMPure $X P)$. Indexing PCR, which adds 8 bp sample-specific indices on both ends of library molecules, was performed using KAPA HiFi HotStart ReadyMix following the manufacturer's protocol for a total of ten PCR cycles. Unfortunately, this was performed using a version of Kapa HiFi that cannot recognize uracils, and therefore the observed ancient DNA-specific substitution rates do not reflect the actual deamination rates in these samples (supplementary table S3 and fig. S4, Supplementary Material online). After indexing PCR, amplified libraries were purified with a combination of $3 \times$ SPRI, silica columns and $1 \times$ SPRI beads until no detectable adapter dimer peak was visible and validated on an Agilent
Bioanalyzer High Sensitivity DNA Analysis chip. All sample libraries were then pooled equimolarly, with extraction blanks pooled at a 1:10 molar ratio compared with sample libraries, and sequenced on one lane of an Illumina HiSeq 2500 System 75 PE with two additional 8 bp index reads.

After sequencing, reads were split into cram files for each library based on matching 8 bp tags, converted into FASTQ files and processed using the ancient DNA analysis pipeline EAGER (Fellows Yates et al. 2021) (nextflow version 20.10.0, EAGER last modified December 2020). The following parameters were used: adapter sequence trimming (forward AGATCGGAAGAGCACACGTCTGAACTCCAGTCACNNNN NNNNATCTCGTATGCCGTCTTCTGCTTG, reverse AGATCG GAAGAGCGTCGTGTAGGGAAAGAGTGTNNNNNNNNGTG TAGATCTCGGTGGTCGCCGTATCATT), aligning to the An. gambiae reference genome (AgamP4) using bwa mem, merging overlapping reads (with default minimum $11 \mathrm{bp}$ overlap), not filtering unmerged reads (longer inserts in younger samples), performing DamageProfiler for a summary of ancient DNA characteristics ( $5^{\prime} \mathrm{C}>\mathrm{T}$ and $3^{\prime} \mathrm{G}>\mathrm{A}$ substitutions, read length in base pairs), removing PCR duplicates and unaligned reads for final bam files. Additionally, samtools coverage was used on the final filtered bam files to get details on nuclear and mitochondrial depth of coverage, as well as the number of covered bases. Final bam files for libraries prepared using different strategies (purification with beads vs columns, unsheared vs sheared) for each of the ten samples were merged, fragments mapping to the mitochondrial genomes were extracted and a consensus mitochondrial genome was created from each sample using bcftools mpileup. Sequencing summary statistics for each library are presented in supplementary table S3, Supplementary Material online.

\section{Testing Different Lysis Conditions on Morphological Damage versus DNA Retrieval}

We next tested different lysis buffer and incubation times on the efficiency of DNA release and the level of morphological damage on different Anopheles species. Samples morphologically designated as An. gambiae $(n=8)$, An. melas $(n=8)$, and $A n$. funestus $(n=26)$ were extracted using three lysis buffers, whose performance was assessed in detail on present-day Anopheles species in Makunin et al. (2021): lysis buffer A (Santos et al. 2018), lysis buffer C (simplified A: $200 \mathrm{mM}$ Tris pH 8.0, $25 \mathrm{mM}$ EDTA pH 8.0, 0.05\% Tween20 and $0.4 \mathrm{mg} / \mathrm{ml}$ Proteinase $\mathrm{K}$ ), and lysis G (simplified [Gutaker et al. 2017]: $10 \mathrm{mM}$ Tris pH 8.0, $10 \mathrm{mM}$ EDTA pH $8.0,5 \mathrm{mM} \mathrm{NaCl}, 0.05 \%$ Tween-20, and $0.4 \mathrm{mg} / \mathrm{ml}$ Proteinase $\mathrm{K})$. Samples were rehydrated $2-3 \mathrm{~h}$ at $37^{\circ} \mathrm{C}$, removed from their respective label pins and submerged in $200 \mu$ l of buffer $\mathrm{A}, \mathrm{C}$, or $\mathrm{G}$ overnight (34 samples) or $2 \mathrm{~h}$ (8 samples) in an oven at $37^{\circ} \mathrm{C}$. Four samples (two An. gambiae and two An. melas) were incubated in lysis buffers $C$ and $G$ without the addition of Proteinase $K$ to assess the level of DNA retrieval and 
morphological damage with minimal tissue clarification. After incubation, lysis buffer was transferred to new tubes, and the samples were ethanol dilution washed $(500 \mu \mathrm{l} 30-50-70 \%$ ethanol, 20 min each) and either stored in 70\% ethanol or fully washed with an additional $90 \%$ and $3 \times 100 \%$ (stored in final $100 \%$ ethanol volume), before returning to the NHM for CPD. Unfortunately, due to a CPD instrument malfunction, there was still substantial morphological damage as a result of the lysis process and/or prolonged desiccation in high ethanol concentrations, which causes tissue collapse.

Lysates were purified using the same MinElute silica column approach, as we confirmed in a ladder experiment using both a short (Thermo Scientific GeneRuler Ultra Low Range DNA Ladder) and long (Thermo Scientific GeneRuler 1 kb Plus Ladder) DNA ladder that we are able to retrieve DNA fragments in the range of $25-10,000 \mathrm{bp}$ using this modified MinElute approach (supplementary fig. S5, Supplementary Material online). DNA extracts were again evaluated using a Quant-iT PicoGreen dsDNA Assay Kit and Agilent Bioanalyzer High Sensitivity DNA Analysis chip or Agilent TapeStation High Sensitivity D5000 ScreenTape System (supplementary table S3 and fig. S6, Supplementary Material online), and a total of $24 \mu$ l was used to prepare libraries. Illumina libraries were prepared using the same NEBNext Ultra II DNA Library Prep Kit following the simplified approach identified in our initial experiment (no prior shearing, purification post-ligation with either $2.5 \times$ or $2.2 \times$ SPRI beads). For indexing PCR, we used the KAPA HiFi HotStart Uracil+ ReadyMix PCR Kit in order to get correct deamination patterns. We used Bioanalyzer or TapeStation concentration values to estimate the optimal number of PCR cycles for each library: An. gambiae complex samples $<10 \mathrm{ng}-12$ cycles, $10-40 \mathrm{ng}-10$ cycles, $>40 \mathrm{ng}-$ 7 cycles; An. funestus all samples-9 cycles. Post-PCR libraries were purified using one round of $1.2 \times$ SPRI beads (An. gambiae complex samples) or two rounds of $1 \times$ SPRI beads (An. funestus samples) to remove the majority of adapter dimers, libraries were checked on an Agilent TapeStation D5000 ScreenTape System, equimolarly pooled into three different pools (An. gambiae and An. melas, An. funestus overnight, An. funestus $2 \mathrm{~h}$ ), again with a smaller proportion for extraction blanks in each, and the pools were sequenced on three lanes of an Illumina HiSeq 4000 System 75 PE with two additional 8 bp index reads. Summary statistics were prepared using EAGER with the same settings as previously described, with An. gambiae and An. melas samples being mapped to AgamP4 and An. funestus samples being mapped to the An. funestus nuclear (AfunF3) and mitochondrial (NC_038158.1) references. After our initial processing and mitochondrial DNA assembly, one of the An. gambiae samples (NHMUK010633485) showed a mitochondrial genome full of $N$ stretches which grouped with other An. funestus samples, and this sample was then mapped to the An. funestus reference instead.
Morphological Damage Assessment across Different Diptera Species

In our final experiment, we examined a wider range of Diptera disease vector species to evaluate the level of morphological damage caused by handling, DNA lysis, and the drying procedure, as well as the amount of DNA that can be retrieved with a $2 \mathrm{~h}$ incubation in the previously tested lysis buffers. For this, we selected 35 samples in total, morphologically designated as Aedes aegypti $(n=9)$, An. gambiae $(n=8)$, Culex pipiens $(n=9)$, and Glossina morsitans $(n=9)$, with three samples each from three different decades (1930s, 1950s and late 1960s/early 1970s defined as 1970s henceforth). Samples were rehydrated for $3 \mathrm{~h}$ at $37^{\circ} \mathrm{C}$, split into three (one for each decade) and incubated in $200 \mu$ of lysis buffer A, C, or $\mathrm{G}$ for $2 \mathrm{~h}$, except for Glossina which due to their size had to be incubated in $1 \mathrm{ml}$ of lysis buffer in a tissue culture plate instead of individual $2.0 \mathrm{ml}$ DNA LoBind tubes. After lysis, all samples were rinsed with $500 \mu \mathrm{l} 30 \%$ ethanol, stored in $500 \mu \mathrm{l} 50 \%$ ethanol, and shipped to the NHM for CPD and imaging. Again, for Glossina samples volume was increased to $1 \mathrm{ml}$ for the first wash and $2 \mathrm{ml}$ storage in a $5.0 \mathrm{ml}$ SafeLock tube due to their size. Lysis buffer was purified using the same modified MinElute silica column method. For Glossina we initially purified only $200 \mu$; however, not enough DNA was detected in this fraction and the remaining $800 \mu$ l were purified and used for quality control assessment instead (increased volumes of PB to $5.6 \mathrm{ml}$ and sodium acetate to $220 \mu \mathrm{l})$. DNA extracts from all four species were evaluated using a Quant-iT PicoGreen dsDNA Assay Kit and Bioanalyzer High Sensitivity DNA Analysis chip or Agilent TapeStation High Sensitivity D5000 ScreenTape System (supplementary table S3 and fig. S10, Supplementary Material online). As the goal of this experiment was primarily to compare morphological damage, we do not present library preparation and sequencing for this experiment.

\section{Supplementary Material}

Supplementary data are available at Genome Biology and Evolution online.

\section{Acknowledgments}

This research was funded by the Wellcome Trust Grant [206194] (which also supports M.K.N.L. and A.M), the London Natural History Museum, and the European Molecular Biology Laboratory. P.K. has been supported by the EMBL-EBI/Wellcome Sanger Institute Post-Doctoral Fellowship Programme (ESPOD). For the purpose of Open Access, the authors have applied a CC BY public copyright licence to any Author Accepted Manuscript version arising from this submission. The authors thank the staff of the Wellcome Sanger Institute Scientific Operations for their contribution to library preparation and sequencing. They are also 
very grateful to members of the Lawniczak and Flicek Research groups for constructive discussions and comments during each step of the project, as well as Alex Ball and Innes Clatworthy (Imaging and Analysis Centre at the NHM core Research Laboratories) for help with the CPD process. And lastly, they are extremely thankful to the entomologists who decades ago collected and determined the Diptera specimens used in this study: Burtt, Buxton, Cattlin, Coetzee, Garnham, Gibson, Gillies, Harbach, Hunt, Knight, Leeson, Lewis, Mackie, Slater, Stymes, Surtees, Sutton, Vincent, and all supporting fieldwork and collection assistants whose names did not fit on specimen labels.

\section{Author Contributions}

P.K., E.M., P.F., and M.K.N.L designed the project. P.K., E.M., and M.M. performed the experiments. P.K., E.M., and A.M. analyzed the data. P.K., E.M., and M.K.N.L. wrote the manuscript with input from M.M, A.M., and P.F.

\section{Data Availability}

Raw sequencing data for all specimen and blank libraries are available under ENA study accession ERP129396.

\section{Literature Cited}

AL-Ahmadi MS. 2019. Pesticides, anthropogenic activities, and the health of our environment safety. In: Larramendy M, Soloneski S, editors. Pesticides-use and misuse and their impact in the environment. London: IntechOpen. p. 73-95. doi: 10.5772/intechopen.84161.

Andrade Justi S, et al. 2021. From e-voucher to genomic data: preserving archive specimens as demonstrated with medically important mosquitoes (Diptera: Culicidae) and kissing bugs (Hemiptera: Reduviidae). PLoS One 16(2):e0247068.

Anopheles gambiae 1000 Genomes Consortium, et al. 2017. Genetic diversity of the African malaria vector Anopheles gambiae. Nature 552:96-100.

Beard CB, Hamm DM, Collins FH. 1993. The mitochondrial genome of the mosquito Anopheles gambiae: DNA sequence, genome organization, and comparisons with mitochondrial sequences of other insects. Insect Mol Biol. 2(2):103-124.

Bi K, et al. 2013. Unlocking the vault: next-generation museum population genomics. Mol Ecol. 22(24):6018-6032.

Briggs AW, et al. 2007. Patterns of damage in genomic DNA sequences from a Neandertal. Proc Natl Acad Sci U S A. 104(37):14616-14621.

Briggs AW, Heyn P. 2012. Preparation of next-generation sequencing libraries from damaged DNA. In: Shapiro B, Hofreiter M, editors. Ancient DNA: methods and protocols. Totowa (NJ): Humana Press. p. 143-154.

Bronner IF, Quail MA. 2019. Best practices for Illumina library preparation. Curr Protoc Hum Genet. 102(1):e86.

Clarkson CS, et al. 2021. The genetic architecture of target-site resistance to pyrethroid insecticides in the African malaria vectors Anopheles gambiae and Anopheles coluzzii. Mol Ecol. 00:1-15. doi: 10.1111/ mec. 15845.

Dabney J, et al. 2013. Complete mitochondrial genome sequence of a Middle Pleistocene cave bear reconstructed from ultrashort DNA fragments. Proc Natl Acad Sci U S A. 110(39):15758-15763.
Edi CAV, et al. 2014. Long-term trends in Anopheles gambiae insecticide resistance in Côte d'Ivoire. Parasit Vectors. 7:500.

Fellows Yates JA, et al. 2021. Reproducible, portable, and efficient ancient genome reconstruction with nf-core/eager. PeerJ 9:e10947.

Fontaine MC, et al. 2015. Mosquito genomics. Extensive introgression in a malaria vector species complex revealed by phylogenomics. Science 347(6217):1258524.

Forgash AJ. 1984. History, evolution, and consequences of insecticide resistance. Pestic Biochem Physiol. 22(2):178-186.

Gansauge M-T, Aximu-Petri A, Nagel S, Meyer M. 2020. Manual and automated preparation of single-stranded DNA libraries for the sequencing of DNA from ancient biological remains and other sources of highly degraded DNA. Nat Protoc. 15(8):2279-2300.

Gilbert MTP, Moore W, Melchior L, Worobey M. 2007. DNA extraction from dry museum beetles without conferring external morphological damage. PLoS One 2(3):e272.

Githeko AK, Service MW, Mbogo CM, Atieli FK. 1996. Resting behaviour, ecology and genetics of malaria vectors in large scale agricultural areas of Western Kenya. Parassitologia 38(3):481-489.

Gloria-Soria A, et al. 2016. Global genetic diversity of Aedes aegypti. Mol Ecol. 25(21):5377-5395.

Gutaker RM, Reiter E, Furtwängler A, Schuenemann VJ, Burbano HA. 2017. Extraction of ultrashort DNA molecules from herbarium specimens. Biotechniques 62(2):76-79.

Hanemaaijer MJ, et al. 2018. Mitochondrial genomes of Anopheles arabiensis, An. gambiae and An. coluzzii show no clear species division. F1000Res. 7:347.

Hua Y-Q, et al. 2016. Sequencing and analysis of the complete mitochondrial genome in Anopheles culicifacies species B (Diptera: Culicidae). Mitochondrial DNA. 27(4):2909-2910.

Jones CM, et al. 2018. Complete Anopheles funestus mitogenomes reveal an ancient history of mitochondrial lineages and their distribution in southern and central Africa. Sci Rep. 8(1):9054.

Kerah-Hinzoumbé C, et al. 2008. Insecticide resistance in Anopheles gambiae from south-western Chad, Central Africa. Malar J. 7:192.

Kleinschmidt I, Rowland M. 2019. 2. Insecticides and malaria. In: Koenraadt CJM, Spitzen J, Takken W, editors. Innovative strategies for vector control. Ecology and control of vector-borne diseases. Vol. 6. Wageningen (Netherlands): Academic Publishers. p. 17-32.

$\mathrm{Li} \mathrm{H}$, et al. 2009. The sequence alignment/map format and SAMtools. Bioinformatics 25(16):2078-2079.

Lindahl T. 1993. Instability and decay of the primary structure of DNA. Nature 362(6422):709-715.

Link V, et al. 2017. ATLAS: analysis tools for low-depth and ancient samples. bioRxiv. 105346. doi: 10.1101/105346.

Liu L, Wu Y, Wei W, Duan X-Y, Qian Z-Q. 2019. The complete mitochondrial genome of the African malaria mosquito Anopheles funestus and its phylogenetic implication. Mitochondrial DNA Part B. 4(1):1065-1067.

Makunin A, et al. 2021. A targeted amplicon sequencing panel to simultaneously identify mosquito species and Plasmodium presence across the entire Anopheles genus. Mol Ecol Resour. 00:1-17. doi: 10.1111/ 1755-0998.13436.

Mendis K, et al. 2009. From malaria control to eradication: the WHO perspective. Trop Med Int Health. 14(7):802-809.

Meyer M, Kircher M. 2010. Illumina sequencing library preparation for highly multiplexed target capture and sequencing. Cold Spring Harb Protoc. 2010(6):pdb.prot5448.

Munywoki DN, Kokwaro ED, Mwangangi JM, Muturi EJ, Mbogo CM. 2021. Insecticide resistance status in Anopheles gambiae (s.l.) in coastal Kenya. Parasit Vectors. 14(1):207.

Oxborough RM, et al. 2015. A new class of insecticide for malaria vector control: evaluation of mosquito nets treated singly with indoxacarb 
(oxadiazine) or with a pyrethroid mixture against Anopheles gambiae and Culex quinquefasciatus. Malar J. 14:353.

Parejo M, Wragg D, Henriques D, Charrière J-D, Estonba A. 2020. Digging into the genomic past of Swiss honey bees by whole-genome sequencing museum specimens. Genome Biol Evol. 12(12):2535-2551.

Parmakelis A, et al. 2008. Historical analysis of a near disaster: anopheles gambiae in Brazil. Am J Trop Med Hyg. 78(1):176-178.

Peng X-Y, Zhou P, Duan X-Y, Qian Z-Q. 2016. The mitochondrial genomes of twelve Anopheles mosquitoes (Diptera: Culicidae) and their phylogenetic implications. Conserv Genet Resour. 8(4):387-390.

Powell JR, Gloria-Soria A, Kotsakiozi P. 2018. Recent history of Aedes aegypti: vector genomics and epidemiology records. Bioscience 68(11):854-860.

Prosser SWJ, deWaard JR, Miller SE, Hebert PDN. 2016. DNA barcodes from century-old type specimens using next-generation sequencing. Mol Ecol Resour. 16(2):487-497.

Racimo F, Renaud G, Slatkin M. 2016. Joint estimation of contamination, error and demography for nuclear DNA from ancient humans. PLoS Genet. 12(4):e1005972.

Riveron JM, et al. 2015. Rise of multiple insecticide resistance in Anopheles funestus in Malawi: a major concern for malaria vector control. Malar J. 14:344.

Rohland N, Glocke I, Aximu-Petri A, Meyer M. 2018. Extraction of highly degraded DNA from ancient bones, teeth and sediments for highthroughput sequencing. Nat Protoc. 13(11):2447-2461.

Santos D, Ribeiro GC, Cabral AD, Sperança MA. 2018. A non-destructive enzymatic method to extract DNA from arthropod specimens: implications for morphological and molecular studies. PLoS One 13(2):e0192200

Satoto TBT, et al. 2019. Insecticide resistance in Aedes aegypti: an impact from human urbanization? PLoS One 14:e0218079.
Skoglund P, et al. 2014. Separating endogenous ancient DNA from modern day contamination in a Siberian Neandertal. Proc Natl Acad Sci U S A. 111(6):2229-2234.

Small ST, et al. 2020. Radiation with reticulation marks the origin of a major malaria vector. Proc Natl Acad Sci $U S$ A. 117(50):31583-31590.

Staats $\mathrm{M}$, et al. 2013. Genomic treasure troves: complete genome sequencing of herbarium and insect museum specimens. PLoS One 8(7):e69189.

Strutzenberger P, Brehm G, Fiedler K. 2012. DNA barcode sequencing from old type specimens as a tool in taxonomy: a case study in the diverse genus Eois (Lepidoptera: Geometridae). PLoS One 7(11):e49710

Timmermans MJTN, Viberg C, Martin G, Hopkins K, Vogler AP. 2016. Rapid assembly of taxonomically validated mitochondrial genomes from historical insect collections. Biol J Linn Soc. 117(1):83-95

Tin MM-Y, Economo EP, Mikheyev AS. 2014. Sequencing degraded DNA from non-destructively sampled museum specimens for RADtagging and low-coverage shotgun phylogenetics. PLoS One 9(5):e96793.

Walker AK, Fitton MG, Vane-Wright RI, Carter DJ. 1999. Insects and other invertebrates. In: Carter D, Walker A, editors. Care and conservation of natural history collections. Oxford: Butterworth Heinemann. p. 37-60.

Weiß CL, et al. 2016. Temporal patterns of damage and decay kinetics of DNA retrieved from plant herbarium specimens. R Soc Open Sci. 3(6): 160239.

Yared S, et al. 2020. Insecticide resistance in Anopheles stephensi in Somali Region, eastern Ethiopia. Malar J. 19:180.

Associate editor: Marta Barluenga 